\title{
PAPER
}

\section{Transverse and longitudinal spin-fluctuations in INVAR $\mathrm{Fe}_{0.65} \mathrm{Ni}_{0.35}$}

To cite this article: J Ross Stewart et al 2019 J. Phys.: Condens. Matter 31025802

View the article online for updates and enhancements.

\section{IOP ebooks}

Bringing you innovative digital publishing with leading voices

to create your essential collection of books in STEM research.

Start exploring the collection - download the first chapter of every title for free. 


\title{
Transverse and longitudinal spin- fluctuations in INVAR $\mathrm{Fe}_{0.65} \mathrm{Ni}_{0.35}$
}

\author{
J Ross Stewart ${ }^{1} \oplus$, Sean R Giblin ${ }^{2}$, Dirk Honecker ${ }^{3}$, Peter Fouquet ${ }^{3}$, \\ Dharmalingam Prabhakaran ${ }^{4}$ and Jonathan W Taylor ${ }^{5}$
}

${ }^{1}$ ISIS Neutron and Muon Facility, Science and Technology Facilities Council, Rutherford Appleton Laboratory, Didcot, OX11 0QX, United Kingdom

2 School of Physics and Astronomy, Cardiff University, Queens Buildings, The Parade, Cardiff, CF24

3AA, United Kingdom

${ }^{3}$ Institut Laue-Langevin, 71 avenue des Martyrs, 38042 Grenoble Cedex 9, France

${ }^{4}$ Clarendon Laboratory, University of Oxford, Parks Road, Oxford, OX1 3PU, United Kingdom

5 ESS Data Management \& Software Centre, Ole Maaløes Vej 3, 2200 Copenhagen N, Denmark

E-mail: ross.stewart@stfc.ac.uk

Received 21 September 2018, revised 26 October 2018

Accepted for publication 6 November 2018

Published 6 December 2018

\begin{abstract}
The presence of spin-fluctuations deep within the ordered state of ferromagnetic INVAR alloy $\mathrm{Fe}_{0.65} \mathrm{Ni}_{0.35}$ has long been suspected but seldom directly observed. Inhomogeneities of one type or another have been cited as important in stabilizing INVAR behaviour-either longitudinal spin-fluctuations associated with the $2 \gamma$-state (local environment) model or transverse magnetisation arising from non-collinear spin structures. In this study we employ small-angle neutron scattering with neutron polarization analysis to distinguish between the two possibilities. Surprisingly we in fact find evidence of dominant but uncorrelated longitudinal spin-fluctuations coexisting with transverse magnetisation which exists in shortrange clusters of size $\sim 130 \AA$. This finding supports recent first principles calculations of $\mathrm{Fe}_{0.65} \mathrm{Ni}_{0.35}$ in which both longitudinal spin-fluctuations and magnetic short-range order are identified as important ingredients in reproducing the equilibrium $\mathrm{Fe}_{0.65} \mathrm{Ni}_{0.35}$ lattice.
\end{abstract}

Keywords: polarized neutron scattering, INVAR effect, small angle neutron scattering

(Some figures may appear in colour only in the online journal)

\section{Introduction}

In 1897 Guillaume established that face-centred-cubic (fcc) alloys of iron and nickel with a concentration of $~ 35$ at.\% nickel exhibit an anomalously small thermal expansion over a wide range of temperature [1]. He considered the expansion of these alloys to be invariable and hence this effect has since become known as the INVAR effect. This effect has since been observed in large number of metal alloys, intermetallics and in some metallic glasses-all of which are magnetically ordered [2]. There is a wide range of applications in which INVAR alloys are used because of this useful property; for example in the manufacture of precision scientific instruments, temperature regulators and microwave resonators. Despite many years of study of the INVAR effect a clear understanding of the mechanism behind this effect is still lacking. INVAR behaviour is clearly related to metallic ferromagnetism $[2,3]$. Below the Curie temperature $\left(T_{C}\right)$ typical coefficients of linear expansion $\left(\alpha_{L}=1 / 3 V \times \mathrm{d} V / \mathrm{d} T\right)$ observed in INVAR materials have a value $\alpha_{L} \simeq 2.1 \times 10^{-6} \mathrm{~K}^{-1}$, while in their respective paramagnetic phases $\alpha_{L}$ increases by around an order of magnitude.

An early attempt at a theoretical description of the INVAR effect is the so-called $2 \gamma$-state model due to Weiss [3]. This model assumes the co-existence of two near degenerate spinstates in f.c.c. iron $(\gamma-\mathrm{Fe})$ : a high spin (HS), high volume state and a low spin (LS), low volume state. Accordingly, thermal spin-excitations from the HS state (labelled $\gamma_{1}$ ) to the LS, $\gamma_{2}$ state leads to a loss of magnetisation on increasing temperature with an associated volume contraction which counteracts phononic thermal expansion. One obvious difficulty with this theory is that while conventional lattice expansion due to 
anharmonic phonons is linear (in the region where the anharmonic terms in the energy are small compared to $k_{\mathrm{B}} T$ ), the thermal population of the HS and LS states should follow a exponential (Boltzmann) temperature dependence, and therefore that cancellation of thermal expansion is not possible over a wide temperature range. This point was well illustrated by Khomskii and Kusmartzev [4], who nevertheless suggested that correlations (magnetic or magneto-elastic) between HS and LS sites might well lead to a more complex temperature dependence.

Electronic band theory calculations confirm the main idea of the $2 \gamma$-state model. First-principles calculations of $\gamma$-Fe, randomly ordered $\mathrm{Fe}_{0.65} \mathrm{Ni}_{0.35}$ and ordered $\mathrm{Fe}_{3} \mathrm{Ni}$ clearly show the existence of two stable magnetic states as described above [5-7]. In particular Entel predicted a change in the relative occupancy between the anti-bonding $t_{2 g}$ majority spin states and the non-bonding $e_{g}$ minority spin states in favour of the latter as the temperature increases [5]. This results in the contraction of bonds, thereby counteracting thermal expansion. However, experimental confirmation of the existence of two spin states at ambient pressure in any INVAR material is, so far, lacking. There is some evidence of closely spaced spinstates in some metallic ferromagnets at high pressure. Recent X-ray magnetic circular dichroism measurements on $\mathrm{Fe}_{72} \mathrm{Pt}_{28}$ revealed that the system undergoes a spin-state transition at an applied pressure of $4 \mathrm{GPa}$ (40 kbar) [8, 9]. Other x-ray diffraction measurements have shown that the non-INVAR metallic ferromagnet $\mathrm{Pd}_{3} \mathrm{Fe}$ exhibits INVAR properties at high pressure [10]. However, precise measurements of the magnetic form factor (magnetisation distribution in $k$-space) in $\mathrm{Fe}_{0.65} \mathrm{Ni}_{0.35}$ INVAR using polarized neutron diffraction [11, 12] in addition to magnetic $x$-ray Compton scattering experiments [13] show that the fraction of unpaired electrons with $\mathrm{e}_{g}$ symmetry remains constant in a range of temperature from $100 \mathrm{~K}-600 \mathrm{~K}$, contradicting the theoretical results of Entel [5] and effectively ruling out the $2 \gamma$-state model as a candidate description of the INVAR effect.

In the mid 1980s, there was an effort by theorists such as Moriya and co-workers to attempt to improve the theoretical description of finite temperature properties of metallic magnets-which are traditionally badly described by simple Stoner models-using self-consistent-renormalisation (SCR) theory [14]. Here, instead of there being two near-degenerate electronic states available to the system, spin fluctuations give rise to a manifold of continuously varying electronic states, resulting in a smoothly varying local magnetisation $\left\langle M_{\mathrm{loc}}^{2}(T)\right\rangle$ which increases monotonically as a function of temperature, with the precise details of the temperature dependence determined by the structure and the occupation of the bands. This temperature variation of $\left\langle M_{\mathrm{loc}}^{2}(T)\right\rangle$ then leads to a magnetovolume effect consistent with INVAR behaviour. This theory received some experimental confirmation from Ishikawa [15] who used neutron scattering to directly observe quasi-elastic magnetic neutron scattering associated with incoherent spinfluctuations in the ordered state of $\mathrm{Fe}_{0.65} \mathrm{Ni}_{0.35}$, although that study did not observe spin-fluctuations in $\mathrm{Fe}_{3} \mathrm{Pt}$.

Further modelling of the temperature dependence of the thermal expansion coefficient from magnetostriction measurements $[16,17]$ using SCR theory showed a remarkable level of agreement, though it was argued-notably by Wohlfarth [18] that the description of the pressure dependence of the Curie temperature in these alloys was less successful. Motivated by the research of the excitations responsible of the INVAR effect, Ishikawa et al performed inelastic neutron scattering measurements on the INVAR alloys $\mathrm{Fe}_{0.65} \mathrm{Ni}_{0.35}$ and $\mathrm{Fe}_{3} \mathrm{Pt}$ and on non-INVAR $\mathrm{Fe}_{50} \mathrm{Ni}_{50}$. They observed that in INVAR $\mathrm{Fe}_{0.65} \mathrm{Ni}_{0.35}$ and $\mathrm{Fe}_{3} \mathrm{Pt}$ spin wave excitations explain only about a half of the temperature decrease of the magnetization while this discrepancy is absent in non-INVAR $\mathrm{Fe}_{50} \mathrm{Ni}_{50}$ $[19,20]$. On the basis of their results they suggested that the variation in amplitude of the local magnetic moment and then the INVAR effect may be due to the presence in these alloys of some hidden (undetected) excitation. Nevertheless, to-date no other source of magnetic excitations which may be responsible for the INVAR effect have been observed. Stoner excitations appear at too high an energy $(\simeq 100 \mathrm{meV})$ to be responsible for the INVAR effect [20-23], while longitudinal spin-fluctuations are observed only in $\mathrm{Fe}_{0.65} \mathrm{Ni}_{0.35}$ and not in $\mathrm{Fe}_{3} \mathrm{Pt}$ [15].

More recent theoretical studies suggest that the INVAR effect is related to thermal magnetic disorder. Two main models of magnetic disorder have been proposed; the disordered local moment (DLM) picture [24-28] and a model incorporating non-collinear magnetic structures [29]. van Schilfgaarde et al find in INVAR concentrations of $\mathrm{Fe}-\mathrm{Ni}$ alloys a magnetic structure characterized, even at zero temperature, by a continuous transition from a ferromagnetic state at high volumes to a disordered non-collinear configuration at low volumes [29]. This non-collinearity gives rise to anomalies in the binding energy volume dependence curve which is directly related to the thermal expansion coefficient through the bulk modulus and Grüneisen constant. Extensive polarized neutron diffraction measurements have been undertaken to look for non-collinear (and hence transverse) magnetism in $\mathrm{Fe}_{0.65} \mathrm{Ni}_{0.35}$ but no sign of non-collinear ferromagnetism is found [30]. However, an indication of the presence of non-collinear moments has been confirmed experimentally at low momentum transfers via polarized small-angle neutron scattering [31] in $\mathrm{Fe}_{0.65} \mathrm{Ni}_{0.35}$. On the basis of this observation Menshikov concluded that in $\mathrm{Fe}_{0.65} \mathrm{Ni}_{0.35}$, non-collinear inhomogeneities are present on a 10-15 $\AA$ length scale. They suggested a model magnetic structure characterised by the occurrence of Fe-rich regions with predominant antiferromagnetic interactions and therefore a low magnetisation. Recent $a b$ initio electronic structure calculations based on the DLM approach, give a good description of the INVAR effect in $\mathrm{Fe}_{0.65} \mathrm{Ni}_{0.35}$ [28], Fe-Pt [27] and $\mathrm{R}-\mathrm{Co}_{2}$ with $\mathrm{R}=\mathrm{Dy}$, Ho [25]. These studies indicate that thermal magnetic disorder (modelled as Ising spin-flips in a local moment picture) leads to INVAR behaviour. However, these models are generally simplistic-assuming fully localised moments, and often localised and randomised defects. More importantly, the DLM picture is lacking experimental justification (beyond the reproduction of the anomalous $\alpha(T)$ behaviour).

In this study, we report on efforts to look for static disorder and transverse magnetism in INVAR $\mathrm{Fe}_{0.65} \mathrm{Ni}_{0.35}$, effectively repeating the measurements of Menshikov [31]. New 
developments in polarized neutron scattering on small-angle neutron scattering (SANS) instruments $[32,33]$ have lead to a resurgence of interest in neutron studies of micromagnetic properties of ferromagnets [34]. We take advantage of these instrumentation and software developments in order to quantitatively characterise the spin-disorder scattering in INVAR - including the ability to distinguish between longitudinal defects akin to the $2 \gamma$-state model and transverse spin-fluctuations expected in DLM and non-collinear state models. Crucially, in our studies we use high purity, optically polished single crystal samples in order to reduce the amount of background nuclear small-angle scattering. We find evidence of extensive transverse and longitudinal static spin-fluctuations in INVAR $\mathrm{Fe}_{0.65} \mathrm{Ni}_{0.35}$, but none at all in non-INVAR $\mathrm{Fe}_{0.5} \mathrm{Ni}_{0.5}$, indicating that spin-disorder is likely to be associated with the INVAR effect. We also present evidence from high-resolution neutron spin-echo spectroscopy of slow dynamical fluctuations at low momentum transfers concomitant with the magnetic spin-disorder scattering seen in SANS, and in broad support of dynamic spin-fluctuation theories.

\section{Methods}

\subsection{Sample preparation}

2.1.1. Polycrystalline $\mathrm{Fe}_{0.65} \mathrm{Ni}_{0.35}$. Polycrystalline samples of $\mathrm{Fe}_{0.65} \mathrm{Ni}_{0.35}$ were prepared by melting appropriate quantities of starting materials with purity of $99.99 \%$ in an argon-arc furnace. The as-melted ingots were then annealed at $800{ }^{\circ} \mathrm{C}$ for $72 \mathrm{~h}$ followed by a slow cool. The stoichiometry of the ingots was verified by performing energy dispersive fluorescence analysis using a commercial scanning electron microscope.

\subsubsection{Single crystal samples of $\mathrm{Fe}_{0.65} \mathrm{Ni}_{0.35}$ and $\mathrm{Fe}_{0.5} \mathrm{Ni}_{0.5}$.} Stoichiometric amounts of high purity (>99.996\%) $\mathrm{Fe}$ and Ni powders where thoroughly mixed inside an argon glove box and loaded into an alumina crucible. The crucible was then sealed inside a $2 \mathrm{~mm}$ wall thickness quartz tube under vacuum. The tube was heated to $1200{ }^{\circ} \mathrm{C}$ and sintered for $3 \mathrm{~d}$. After confirming the single phase purity the cylindrical shaped sintered rod was loaded in an optical floatingzone furnace. To increase the density of the sintered rod it was melted at a faster growth rate of $10 \mathrm{~mm} \mathrm{~h}^{-1}$ under purified argon atmosphere. Finally, pre-melted rods of $\mathrm{Fe}_{0.65} \mathrm{Ni}_{0.35}$ and $\mathrm{Fe}_{0.5} \mathrm{Ni}_{0.5}$ were used to grow a crystals at a growth rate of $2.5 \mathrm{~mm} \mathrm{~h}^{-1}$ with $20 \mathrm{rpm}$ counter rotation of the feed and seed rods respectively under purified argon flow of $11 \mathrm{~min}^{-1}$.

$\mathrm{M}-\mathrm{H}$ curves measured on single crystal $\mathrm{Fe}_{0.65} \mathrm{Ni}_{0.35}$ up to $2 \mathrm{~T}$ were in good agreement with previously published studies $[35,36]$ with magnetic saturation at fields $>0.2 \mathrm{~T}$ and a saturation moment of $1.22 \mu_{\mathrm{B}}$ per atom agreeing well with the published value at room temperature of $1.28 \mu_{\mathrm{B}}$ [37].

\subsection{Small-angle neutron scattering [38, 39]}

Two experiments using SANS with neutron polarization analysis on the D33 SANS instrument at the Institut

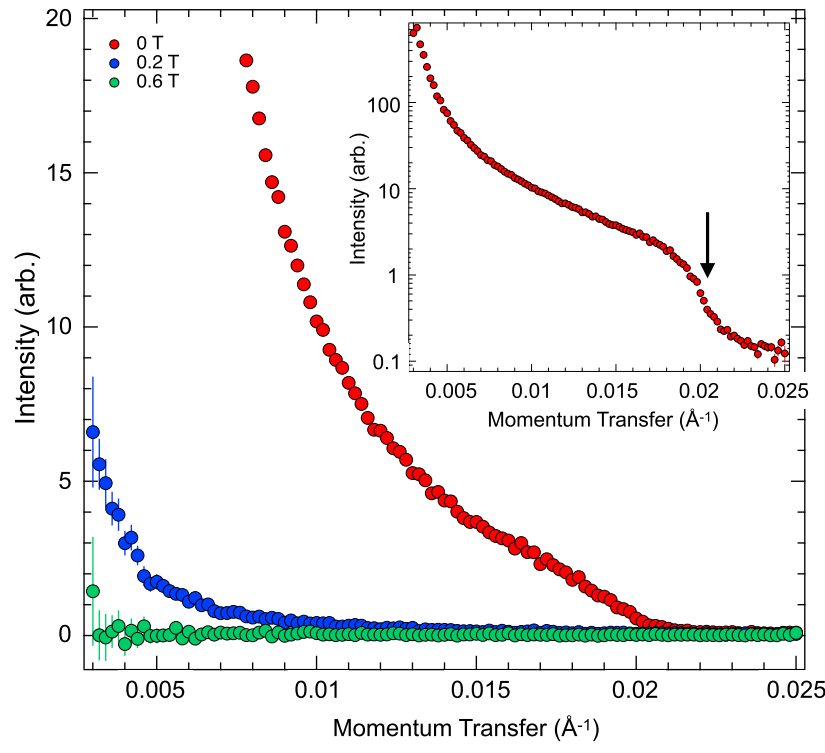

Figure 1. Radially averaged SANS in $\mathrm{Fe}_{0.65} \mathrm{Ni}_{0.35}$ as a function of field. The SANS measured at $2 \mathrm{~T}$ has been subtracted. By $0.6 \mathrm{~T}$ the magnetic domain SANS is suppressed and the sample is single domain. The inset shows unsubtracted data taken at zero field with the arrow indicating the spin-wave scattering cut-off.

Laue-Langevin (ILL) [40] were performed on highly polished single crystals of INVAR $\mathrm{Fe}_{0.65} \mathrm{Ni}_{0.35}$ and non-INVAR $\mathrm{Fe}_{0.5} \mathrm{Ni}_{0.5}$. The crystals were disk-shaped with a diameter of $\sim 12 \mathrm{~mm}$ and a thickness of between $\sim 1 \mathrm{~mm}$ and $\sim 3 \mathrm{~mm}$ (in the first experiment [38] the crystals were thicker). The polarized neutron beam was incident on the flat polished faces of the single crystals, which were approximately normal to the $\{001\}$ crystal axes. The choice of highly polished single crystals was made to reduce the SANS signal due to surface roughness and grain boundaries to a minimum. A variable magnetic field of between $0 \mathrm{~T}$ and $2 \mathrm{~T}$ was applied to the sample in the plane of the disk-shaped crystals, transverse to the beam direction. This resulted in small demagnetisation factors which were estimated to be of the order of $N \lesssim 0.1$ for all samples and therefore neglected. The SANS and magnetic field geometry define a natural orthogonal coordinate set (following the convention of Moon et al [41]) which was later used in the data reduction and analysis; the incident neutron wavevector $\mathbf{k}_{i}$ defines the $x$-direction, while the magnetic field is applied along the $z$-direction (horizontal and transverse to $\mathbf{k}_{i}$ ). The SANS detector is therefore in the $y-z$ plane and so accessible momentum transfers in the experiment are limited to that plane. Experiments were performed in both polarized and unpolarized beam mode on D33. This was achieved via the insertion of a supermirror polarizer of known efficiency. Analysis of the scattered neutron polarization was provided using a single-crystal silicon windowed ${ }^{3} \mathrm{He}$ spin-filter [42]. This was shielded from the magnetic fields applied at the sample position using a 'magic box' which contained an integrated adiabatic-fast-passage ${ }^{3} \mathrm{He}$ flipper [43]. All the SANS experiments were performed using a neutron wavelength of $6 \AA$. Data visualisation, reduction and correction was performed using the ILL LAMP suite of programs [44]. 


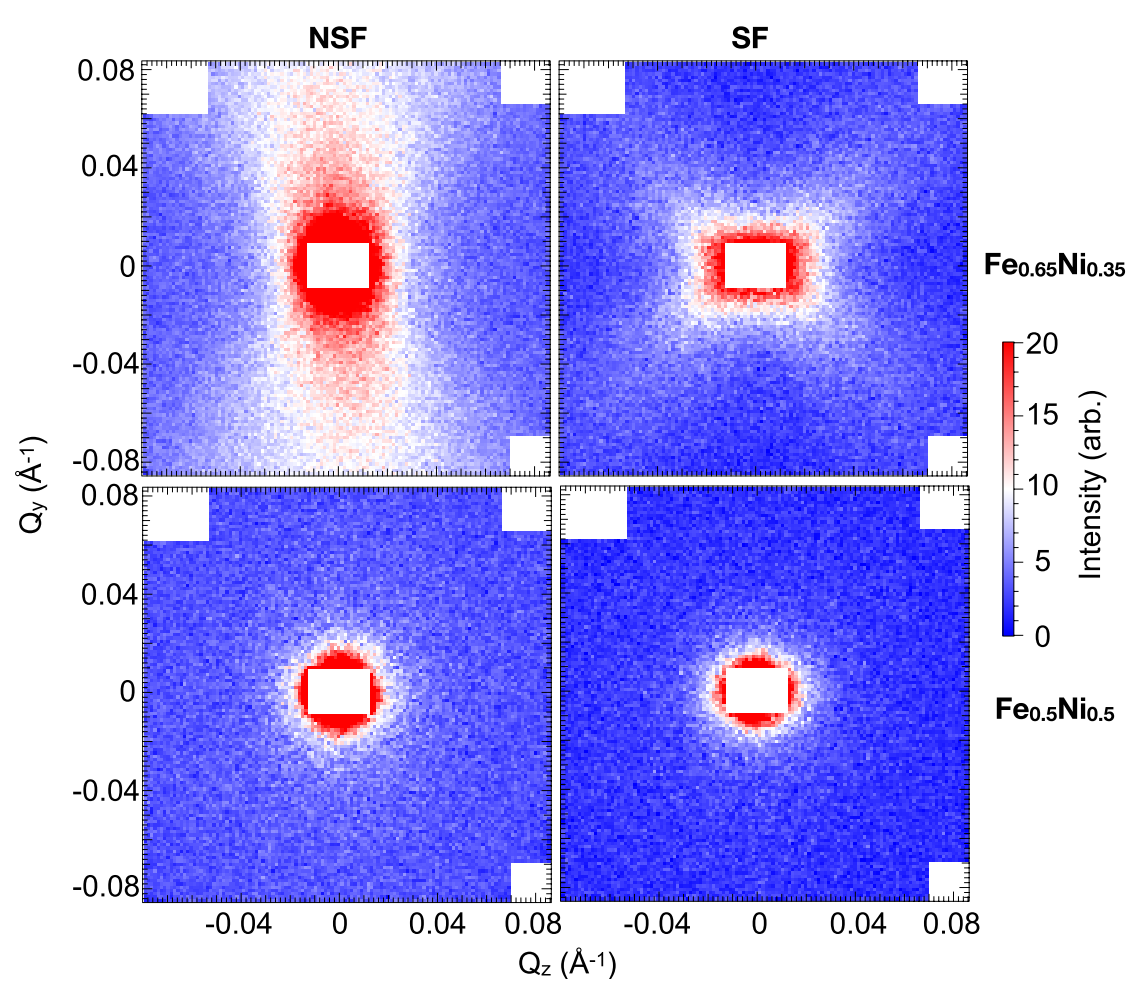

Figure 2. SANS with polarization analysis at $300 \mathrm{~K}$ and $0.6 \mathrm{~T}$ for INVAR $\mathrm{Fe}_{0.65} \mathrm{Ni}_{0.35}$ (top row) and non-INVAR $\mathrm{Fe}_{0.5} \mathrm{Ni}_{0.5}$ (bottom row). The left column shows the average non-spin-flip cross-section and the right column shows the spin-flip cross-section. Masked white regions in the corners of the scattering patterns denote areas of the detector not covered by the ${ }^{3} \mathrm{He}$ spin-filter analyser.

\subsection{Ferromagnetic neutron-spin-echo (FMNSE) [45]}

FMNSE measurements were carried out on a polycrystalline ingot of $\mathrm{Fe}_{0.65} \mathrm{Ni}_{0.35}$ with a mass of $\sim 100 \mathrm{~g}$ using the spinecho spectrometer IN11 at the ILL [46]. A saturating vertical field of $1 \mathrm{~T}$ was applied to align the magnetic domains in the sample and hence preserve the neutron polarization. The temperature dependence of the intermediate scattering function $S(q, t) / S(q, 0)$ was measured using neutron wavelength of $\lambda=5.5 \AA$ at a scattering angle $2 \theta=4^{\circ}$. Data collection, reduction and visualisation was performed using the IN11 Igor Pro (WaveMetrics, Inc.) suite of programs.

\section{Results}

\subsection{Unpolarized SANS}

In order to determine the field dependence of the magnetic domain scattering in INVAR we measured SANS at several fields between $0 \mathrm{~T}$ and $2 \mathrm{~T}$. The measured SANS for $0 \mathrm{~T}, 0.2$ $\mathrm{T}$ and $0.6 \mathrm{~T}$ is presented in figure 1. The SANS measured at $2 \mathrm{~T}$ (where $\mathrm{Fe}_{0.65} \mathrm{Ni}_{0.35}$ is known to be single domain) has been subtracted from the data. The $2 \mathrm{~T}$ dataset will include all nondomain scattering such as nuclear background and residual magnetic scattering. The plot shows the suppression of magnetic domain scattering with increasing field, indicating that at $0.6 \mathrm{~T} \mathrm{Fe}_{0.65} \mathrm{Ni}_{0.35}$ is single domain. Any spin-misalignment scattering measured at or above $0.6 \mathrm{~T}$ therefore must be due to intrinsic non-collinear spins and not to domains.

The zero-field SANS data (shown in the inset of figure 1) shows a sharp rise at around $q=0.02 \AA^{-1}$, corresponding to a scattering angle of $2 \theta=0.55^{\circ}$ for neutrons of wavelength $\lambda=6 \AA$. We attribute this feature to the onset of spin-wave scattering at low momentum-transfers. For ferromagnets with quadratic spin-wave dispersion, neutron kinematics dictate that spin-wave scattering can only be detected below a certain cut-off angle,

$$
\sin \theta_{c}=\frac{\hbar^{2}}{2 m_{\mathrm{n}} D}
$$

where $m_{\mathrm{n}}$ is the mass of the neutron and $D$ is the spin-wave stiffness, $\hbar \omega=D q^{2}$. According to the work of Hatherly [47] $D \simeq 200 \mathrm{meV} \AA^{2}$ for $\mathrm{Fe}_{0.65} \mathrm{Ni}_{0.35}$, corresponding to a cutoff angle of $\theta_{c}=0.6^{\circ}$, in good agreement with the observed cut-off in this experiment. Henceforth, in order to eliminate contributions to the SANS from spin-waves, we restrict ourselves to scattering at angles $2 \theta \geqslant 0.6^{\circ}$. A similar magnetic field dependence of the unpolarized SANS signal was seen in $\mathrm{Fe}_{0.5} \mathrm{Ni}_{0.5}$ with a similar spin-wave cut-off.

\subsection{SANS with polarization analysis}

Having determined that $0.6 \mathrm{~T}$ was sufficient to produce a single magnetic domain in our $\mathrm{Fe}_{0.65} \mathrm{Ni}_{0.35}$ and $\mathrm{Fe}_{0.5} \mathrm{Ni}_{0.5}$ samples, we proceeded to perform SANS with polarization analysis on both samples at a fixed field of 0.6 T. The standard uniaxial polarization measurements were taken with the polarization aligned alternately parallel and anti-parallel to the applied field along the $z$-direction, and then analysed parallel and anti-parallel. This procedure results in the measurement of four cross-sections; the non-spin-flip (NSF) 

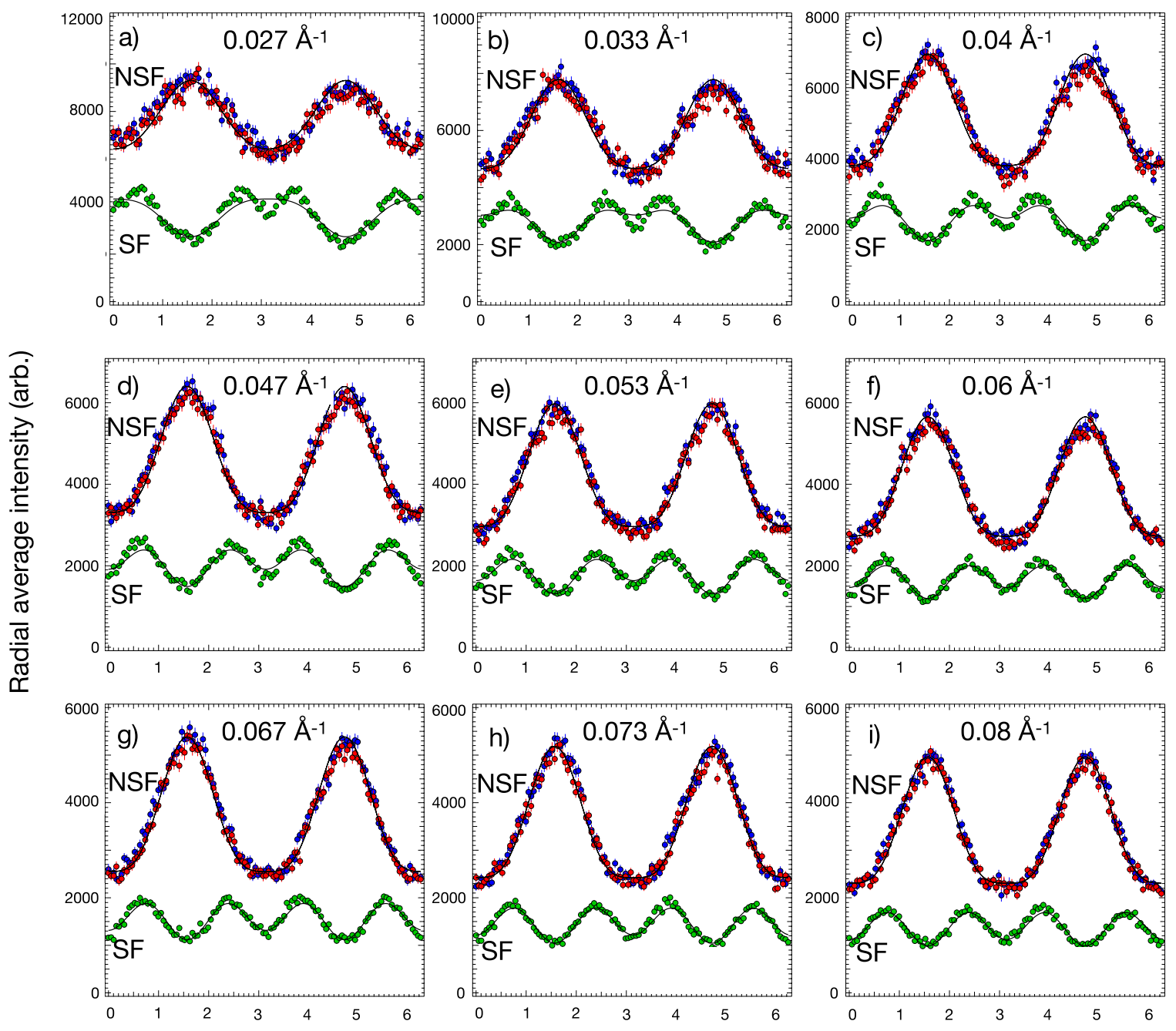

Azimuthal angle (rad)

Figure 3. Radially averaged plots at constant $|q|$ of the NSF (blue: $(\mathrm{d} \Sigma / \mathrm{d} \Omega)^{++}$, red: $(\mathrm{d} \Sigma / \mathrm{d} \Omega)^{--}$) and average SF (green) SANS from $\mathrm{Fe}_{0.65} \mathrm{Ni}_{0.35}$ at $300 \mathrm{~K}$ and $0.6 \mathrm{~T}$, plotted agains the azimuthal angle $\phi$. Plots (a)-(i) show the SANS at $0.027 \leqslant|q| \leqslant 0.08$. The solid lines through the data are fits to equation (2).

cross-sections $(\mathrm{d} \Sigma / \mathrm{d} \Omega)^{++}$and $(\mathrm{d} \Sigma / \mathrm{d} \Omega)^{--}$, and the spinflip (SF) cross-sections $(\mathrm{d} \Sigma / \mathrm{d} \Omega)^{+-}$and $(\mathrm{d} \Sigma / \mathrm{d} \Omega)^{-+}$. Here the + and - superscripts refer to polarization parallel and anti-parallel respectively to the applied field, with the first/ second superscripts referring to the initial/final polarization direction. It was empirically observed for both samples that the two non-spin-flip and the two spin-flip cross-sections were identical. This observation implies that terms in the scattering intensity due to nuclear-magnetic interference are zero (since the sign of these terms depend on the direction of incident polarization) [33]. Furthermore, the lack of magnetic anisotropy (shape or otherwise in our samples) suggests that any transverse magnetization in the sample (along $y$ ) should be isotropic. Under these conditions the polarized neutron crosssections can be written as $[33,48]$,

$$
\begin{aligned}
\frac{\mathrm{d} \Sigma^{\mathrm{NSF}}}{\mathrm{d} \Omega} & =N^{2}(q)+M_{y}^{2}(q) \sin ^{2} \phi \cos ^{2} \phi+M_{z}^{2}(q) \sin ^{4} \phi \\
\frac{\mathrm{d} \Sigma^{\mathrm{SF}}}{\mathrm{d} \Omega} & =M_{x}^{2}(q)+M_{y}^{2}(q) \cos ^{4} \phi+M_{z}^{2}(q) \sin ^{2} \phi \cos ^{2} \phi
\end{aligned}
$$

where $\phi$ is the azimuthal angle in the $y-z$ plane between the $z$-axis and the scattering vector $q . N(q)$ and $M(q)=\left[M_{x}(q), M_{y}(q), M_{y}(q)\right]$ are the Fourier transforms of the nuclear and magnetic scattering length density respectively, with $M_{x}(q)$ and $M_{y}(q)$ representing the transverse magnetization and $M_{z}(q)$ the longitudinal magnetization. Again - based on the assumption of magnetic isotropy in the sample-we assume that all $q$-dependence of the terms in the cross-section is isotropic at small angles and that there is no azimuthal dependence of the individual magnetization Fourier components.

The polarized SANS scattering for INVAR $\mathrm{Fe}_{0.65} \mathrm{Ni}_{0.35}$ and non-INVAR $\mathrm{Fe}_{0.5} \mathrm{Ni}_{0.5}$ measured at room temperature and $0.6 \mathrm{~T}$ are shown in figure 2. On initial inspection of the azimuthal dependance of the data, it is clear that there is significant spin-misalignment scattering in $\mathrm{Fe}_{0.65} \mathrm{Ni}_{0.35}$ but not in $\mathrm{Fe}_{0.5} \mathrm{Ni}_{0.5}$. This suggests that magnetic inhomogeneity is somehow enhanced at the critical INVAR concentration in agreement with the observations of Menshikov [31]. The residual small angle scattering at low $q$ seen in both 


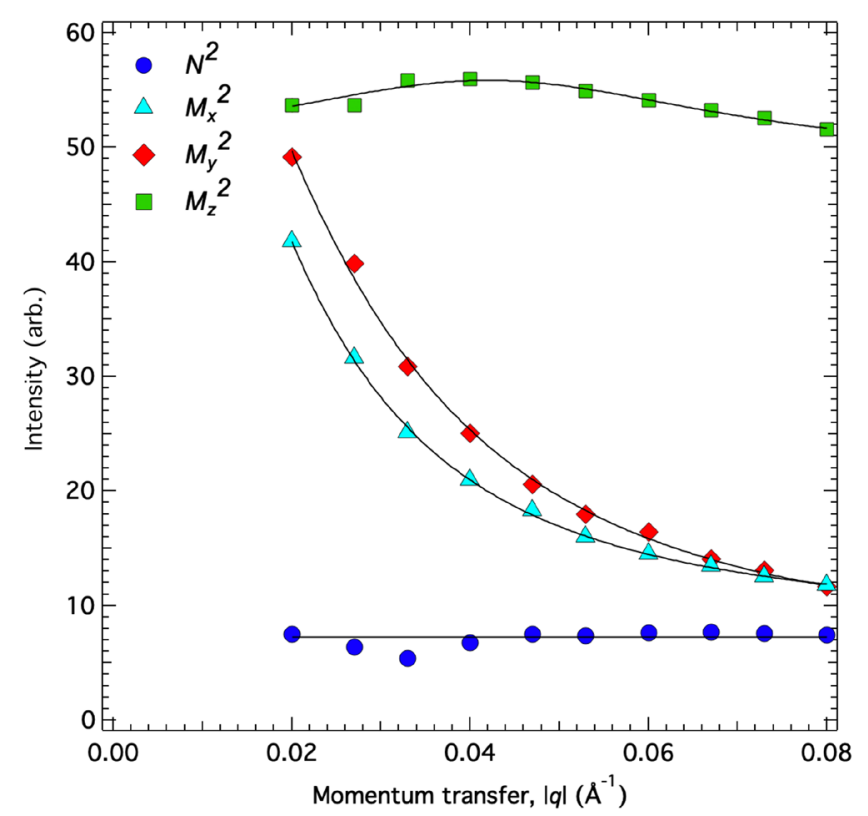

Figure 4. Individual SANS components, nuclear $N^{2}(q)$ (blue circles), transverse magnetic $M_{x}^{2}(q)$ (cyan triangles) and $M_{z}^{2}(q)$ (red diamonds) and longitudinal magnetic $M_{z}^{2}(q)$ (green squares) measured on $\mathrm{Fe}_{0.65} \mathrm{Ni}_{0.35}$ at $300 \mathrm{~K}$ and $0.6 \mathrm{~T}$ extracted using equation (2). The lines are fits to a Lorentzian form.

$\mathrm{Fe}_{0.65} \mathrm{Ni}_{0.35}$ and $\mathrm{Fe}_{0.5} \mathrm{Ni}_{0.5}$ is likely due to spin-waves below $|q|=0.02 \AA^{-1}$ and possible background contributions from the sample holder. By inspection and comparison with equation (2), it is clear that the dominant cause of magnetic scattering (for $|q|>0.03 \AA^{-1}$ ) is longitudinal spin-fluctuations associated with the z-component of magnetization, $M_{z}(q)$.

Some checks of the field and temperature dependence of the SANS in $\mathrm{Fe}_{0.65} \mathrm{Ni}_{0.35}$ were performed. Firstly, increasing the field to $1.5 \mathrm{~T}$ (which was the maximum possible consistent with the operation of the ${ }^{3} \mathrm{He}$ spin-filter) had little discernible effect on the SANS beyond some suppression of the low $q$ $\left(<0.02 \AA^{-1}\right)$ spin-wave scattering. There was also very little temperature dependence of the SANS between $250 \mathrm{~K}$ and $320 \mathrm{~K}$ (in the region of minimal thermal expansion). However, at low temperatures $(40 \mathrm{~K})$ the intensity of the magnetic scattering (both transverse and longitudinal components) was reduced by around a factor of two-in good agreement with previous studies [31].

In order to apply a quantitative analysis of the data, radial averages at fixed $|q|$ were extracted from the background subtracted data and the NSF and SF cross-sections fitted to equation (2) simultaneously. Example fits of the radially averaged data are shown in figure 3 .

Figure 4 shows plots of the extracted components of the polarized SANS measurements extracted from the fits to equation (2). We can see, firstly, that the nuclear small-angle scattering is indeed small and flat in the $\mathrm{Fe}_{0.65} \mathrm{Ni}_{0.35}$ single crystal sample, indicating the quality of the crystal and the scattering surface. The magnetic components of the scattering are dominated by a large $M_{z}$ longitudinal component which is flat as a function of $|q|$ indicating uncorrelated fluctuations. By contrast the two transverse components of magnetization

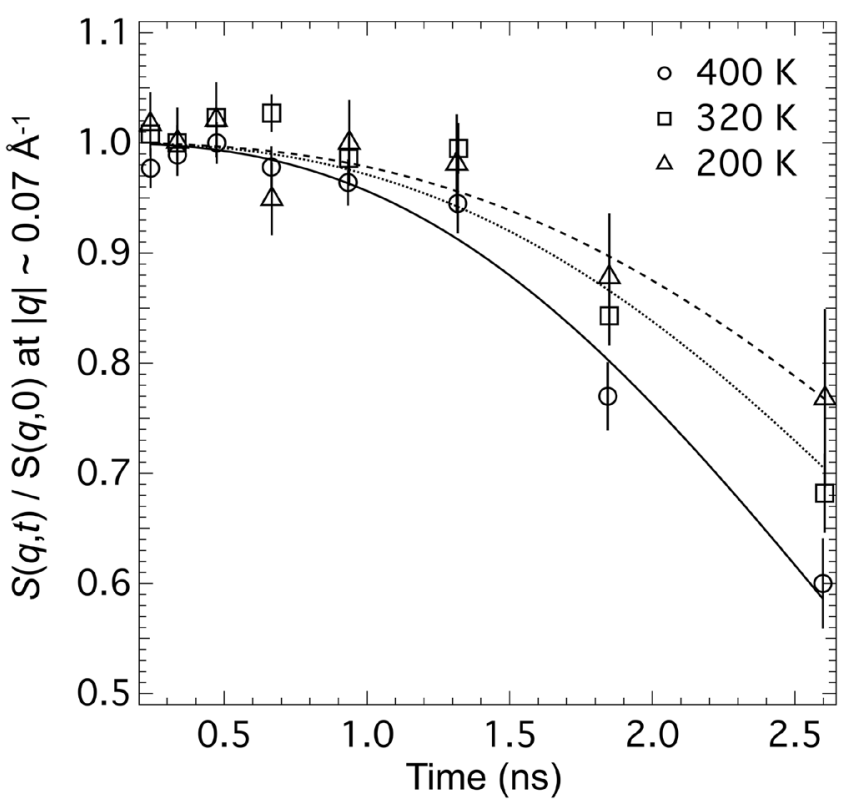

Figure 5. The normalised intermediate scattering function $S(q, t) / S(q, 0)$ with $|q| \simeq 0.07 \AA^{-1}$ measured on $\mathrm{Fe}_{0.65} \mathrm{Ni}_{0.35}$ using the IN11 spin-echo spectrometer as a function of temperature. These show evidence of slow spin-fluctuations in the ordered ferromagnetic state. Lines are fits to a stretched exponential decay in order to loosely quantify the relaxation time.

$M_{x}$ and $M_{y}$ display a similar Lorentzian dependence on $|q|$ indicating Ornstein-Zernicke correlated clusters [49] of range $\xi \sim 130 \AA$.

\subsection{FMNSE measurements}

The intermediate scattering function $S(q, t) / S(q, 0)$ with $|q| \simeq 0.07 \AA^{-1}$ measured from our FMNSE study is plotted in figure 5 for three temperatures; $200 \mathrm{~K}, 320 \mathrm{~K}$ and $400 \mathrm{~K}$. The advantage of spin-echo neutron spectroscopy over conventional neutron spectroscopy is that the technique directly measures the time-dependent spin-spin autocorrelation function $S(t)$ (see for example the review of Ehlers [50]). In the FMNSE configuration, IN11 is able to measure over approximately one decade of time between 0.25 and $2.6 \mathrm{~ns}$, and is therefore sensitive to spin-fluctuations on that timescale. The data shown in figure 5 were corrected for instrumental resolution by dividing the data using a low temperature run measured at $5 \mathrm{~K}$. In figure 5 we see that $S(t)$ starts to decrease at long Fourier times above $\sim 1 \mathrm{~ns}$ indicating the presence of nanosecond timescale spin-fluctuations in $\mathrm{Fe}_{0.65} \mathrm{Ni}_{0.35}$. While this is in the upper reaches of the available time range on IN11, it is within the time-resolution of the instrument, and we note that the characteristic spin-fluctuation rate increases systematically with temperature. In order to give a quantitative estimate of the timescale of the observed spin-fluctuations, the data were fitted to a stretched exponential function, $S(t) / S(0)=\exp \left[-(t / \tau)^{\beta}\right]$. We find relaxation times $\tau^{\beta}$ of $\sim 22 \mathrm{~ns}, \sim 34 \mathrm{~ns}$ and $\sim 45 \mathrm{~ns}$ at $400 \mathrm{~K}, 320 \mathrm{~K}$ and $200 \mathrm{~K}$ respectively. Since these spin-fluctuations are measured at a momentum transfer of $|q| \simeq 0.07 \AA^{-1}$ they correspond to a 
similar length scale as the spin-fluctuations measured in the polarized SANS measurement. It is important to note that the fluctuation rates measured here are well within the kinematic range of the SANS experiment. While the statistical quality of the measured $S(t)$ does not permit a robust fit, the observed line-shape is evidently strongly non-exponential in form.

\section{Discussion}

The presence of significant longitudinal and transverse spinfluctuations in INVAR $\mathrm{Fe}_{0.65} \mathrm{Ni}_{0.35}$ unambiguously confirms the presence of magnetic inhomogeneities and non-collinear components of the magnetisation deep in the FM ordered state. This is consistent with the observations of a large highfield susceptibility in the ordered state $[35,36]$ and also in qualitative agreement with previous polarized neutron SANS and neutron depolarization in transmission measurements of the group of Grigoriev et al $[51,52]$. The lack of spin-fluctuations in $\mathrm{Fe}_{0.5} \mathrm{Ni}_{0.5}$ is also consistent with previous studies and points to a likely connection between these fluctuations and the INVAR effect.

In a sense, these experiments are then consistent with either a longitudinal spin-fluctuation model (i.e. the $2 \gamma$-state model) or a non-collinear spin (DLM) model or both, since both types of fluctuations are seen in SANS. But we can state that neither of these models in isolation can explain the presence of both types of spin-fluctuation. Additionally, the observation of some temperature dependence of the spin-fluctuations, both from the polarized SANS data and the FMNSE experiment suggests that spin-dynamics of the magnetic inhomogeneities in $\mathrm{Fe}_{0.65} \mathrm{Ni}_{0.35}$ may have a significant role to play in the INVAR effect in these materials. This is in, at least qualitative, agreement with the spin-fluctuation theories of Moriya and others $[14,15]$ in which the temperature dependence of the spin-fluctuation amplitude is a key ingredient for INVAR behaviour.

It is interesting that the longitudinal spin-fluctuations appear to be uncorrelated in space implying these cannot be associated with previously observed atomic short-range order scattering in $\mathrm{Fe}_{0.65} \mathrm{Ni}_{0.35}[53,54]$. It is tempting, however, to associate the transverse spin-fluctuations seen in this study with shear-wave type deformations in $\mathrm{Fe}_{0.65} \mathrm{Ni}_{0.35}$ attributed to a large magneto-volume effect-particularly since the observed length scales of these (between 20-50 $\AA$ ) is not too dissimilar with the length scales that we observe of $\sim 130 \AA$ [54].

Very recent first-principles modelling of $\mathrm{Fe}_{0.65} \mathrm{Ni}_{0.35}$ using the so-called spin-wave method [55] have highlighted the importance of accounting for both transverse spin-fluctuations (magnetic short-range order) and longitudinal spin-fluctuations in the calculations in order to arrive at an accurate model of the $\mathrm{Fe}_{0.65} \mathrm{Ni}_{0.35}$ lattice and that at elevated temperatures, the extra magnetic entropy associated with these inhomogeneities contributes noticeably to the equilibrium lattice constant. The spin-fluctuations we have observed here are fully consistent with this model.

\section{Acknowledgments}

The authors would like to thank Giovanni Pasquino and Toby Willis (both formerly at Royal Holloway University of London) for help with the neutron experiments. Valuable technical support was provided by Michel Bonnaud and Eric Thaveron of the ILL. Polarized ${ }^{3} \mathrm{He}$ spin-filters on D33 were provided by David Jullien, Pascale Mouveau and Antonello Rizzo. JRS would like to acknowledge valuable discussions with Julie Staunton, Katia Pappas and Jon Goff. DP gratefully acknowledges financial support from EPSRC, grant numbers EP/K028960/1 and EP/M020517/1.

\section{ORCID iDs}

J Ross Stewart (1) https://orcid.org/0000-0003-0053-0178

\section{References}

[1] Guillaume C É 1897 C. R. Acad. Sci. 125 235-8

[2] Wassermann E F 1990 Handbook of Ferromagnetic Materials vol 5 (Amsterdam: Elsevier) pp 237-322

[3] Weiss R J 1963 Proc. Phys. Soc. 82 281-8

[4] Khomskii D I and Kusmartsev F V 2004 Phys. Rev. B 70 012413-4

[5] Entel P, Hoffmann E, Mohn P, Schwarz K and Moruzzi V L 1993 Phys. Rev. B 47 8706-20

[6] Kaspar J and Salahub D R 1981 Phys. Rev. Lett. 4754

[7] Williams A R, Moruzzi V L and Gelatt C D Jr 1983 J. Magn. Magn. Mater. 31-34 88-94

[8] Odin S, Baudelet F, Giorgetti C, Dartyge E, Itié J P, Polian A, Chervin J C, Pizzini S, Fontaine A and Kappler J P 1999 Europhys. Lett. 47378

[9] Odin S, Baudelet F, Dartyge E, Itié J P, Polian A, Chervin J C, Kappler J P, Fontaine A and Pizzini S 2000 Phil. Mag. B 80 155-63

[10] Winterrose M, Lucas M S, Yue A, Halevy I, Mauger L, Muñoz J, Hu J, Lerche M and Fultz B 2009 Phys. Rev. Lett. 102237202

[11] Ito Y, Akimitsu J, Matsui M and Chikazumi S 1979 J. Magn. Magn. Mater. 10 194-6

[12] Brown P J, Neumann K U and Ziebeck K R A 2001 J. Phys.: Condens. Mater. 13 1563-9

[13] Taylor J W, Duffy J A, Bebb A, McCarthy J, Lees M R, Cooper M J and Timms D 2002 Phys. Rev. B 65224408

[14] Moriya T and Usami K 1980 Solid State Commun. 34 95-9

[15] Ishikawa Y, Onodera S and Tajima K 1981 Solid State Commun. 38 561-4

[16] Ono F 1981 J. Phys. Soc. Japan 50 2231-5

[17] Yamada O 1983 Physica B 119 90-5

[18] Wohlfarth E P 1980 Solid State Commun. 35 797-800

[19] Ishikawa Y, Onodera S and Tajima K 1979 J. Magn. Magn. Mater. 10 183-90

[20] Onodera S, Ishikawa Y and Tajima K 1981 J. Phys. Soc. Japan 50 1513-22

[21] Tajima K, Böni P, Shirane G, Ishikawa Y and Kohgi M 1987 Phys. Rev. B 35 274-8

[22] Ishikawa Y, Noda Y, Ziebeck K R A and Givord D 1986 Solid State Commun. $\mathbf{5 7}$ 531-4

[23] Ishikawa Y, Kohgi M, Onodera S and Grier B H 1986 Solid State Commun. $\mathbf{5 7}$ 535-8

[24] Akai H and Dederichs P H 1993 Phys. Rev. B 47 8739-47 
[25] Khmelevskyi S and Mohn P 2002 Phys. Rev. B 66220404

[26] Crisan V, Entel P, Ebert H, Akai H, Johnson D D and Staunton J B 2002 Phys. Rev. B 66014416

[27] Khmelevskyi S, Turek I and Mohn P 2003 Phys. Rev. Lett. 91037201

[28] Liot F and Hooley C A 2012 (arXiv:1208.2850v1)

[29] van Schilfgaarde M, Abrikosov I A and Johansson B 1999 Nature 400 46-9

[30] Cowlam N and Wildes A R 2003 J. Phys.: Condens. Mater. 15 1-11

[31] Menshikov A Z and Schweizer J 1996 Solid State Commun. $100251-5$

[32] Dewhurst C D 2008 Meas. Sci. Technol. 19034007

[33] Honecker D, Ferdinand A, Döbrich F, Dewhurst C D, Wiedenmann A, Gómez-Polo C, Suzuki K and Michels A 2010 Eur. Phys. J. B 76 209-13

[34] Michels A 2014 J. Phys.: Condens. Matter 26383201

[35] Crangle J and Hallam G C 1963 Proc. R. Soc. Lond. A 272 119-32

[36] Shiga M 1967 J. Phys. Soc. Japan 22 539-46

[37] Rancourt D G, Hargraves P, Lamarche G and Dunlap R A 1990 J. Magn. Magn. Mater. 87 71-82

[38] Stewart J R, Giblin S R, Taylor J W, Willis T J and Honecker D 2014 Small angle neutron scattering investigation of transverse magnetisation in INVAR $\mathrm{Fe}_{0.65} \mathrm{Ni}_{0.35}$ (Institut Laue-Langevin (ILL)) (https://doi.ill.fr/10.5291/ ILL-DATA.5-42-364)

[39] Stewart J R, Giblin S R and Honecker D 2016 Measurements of transverse and longitudinal 'static' spin fluctuations in INVAR (Institut Laue-Langevin (ILL)) (https://doi.ill. fr/10.5291/ILL-DATA.5-42-421)

[40] Dewhurst C D, Grillo I, Honecker D, Bonnaud M, Jacques M, Amrouni C, Perillo-Marcone A, Manzin G, Cubitt R and IUCr 2016 J. Appl. Cryst. 49 1-14
[41] Moon R M, Riste T and Koehler W C 1969 Phys. Rev. $181920-31$

[42] Andersen K H, Cubitt R, Humblot H and Jullien D 2006 Physica B 385-6 1134-7

[43] Petoukhov A K et al 2006 Nucl. Instrum. Methods Phys. Res. A $560480-4$

[44] Richard D, Ferrand M and Kearley G J 1996 J. Neutron Res. $433-9$

[45] Taylor J W, Stewart J R, Pasquino G and Fouquet P 2012 Ferromagnetic-NSE study of the low-frequency spin-dynamical signature of the INVAR effect in $\mathrm{Fe}_{0.65}$ $\mathrm{Ni}_{035}$ (Institut Laue-Langevin (ILL)) (https://doi.ill. fr/10.5291/ILL-DATA.4-01-1111)

[46] Dagleish P, Hayter J B and Mezei F 1980 Neutron Spin Echo (Lecture Notes in Physics vol 128) (Berlin: Springer) pp 66-71

[47] Hatherly M, Hirakawa K, Lowde R D, Mallett J F, Stringfellow M W and Torrie B H 1964 Proc. Phys. Soc. 84 55-62

[48] Michels A and Weissmüller J 2008 Rep. Prog. Phys. 71 066501-42

[49] Collins M R 1989 Magnetic Critical Scattering (Oxford: Oxford University Press)

[50] Ehlers G 2006 J. Phys.: Condens. Matter 18 R231-44

[51] Grigoriev S V, Maleyev S V, Okorokov A I, Eckerlebe H and van Dijk N H 2004 Phys. Rev. B 69134417

[52] Grigoriev S V, Maleyev S V, Okorokov A I and Runov V 1998 Phys. Rev. B 58 3206-11

[53] Tsunoda Y, Hao L, Shimomura S, Ye F, Robertson J L and Fernandez-Baca J 2008 Phys. Rev. B 78 094105-8

[54] Robertson J L, Ice G, Sparks C J, Jiang X, Zschack P, Bley F, Lefebvre S and Bessiere M 1999 Phys. Rev. Lett. 82 2911-4

[55] Ruban A V 2017 Phys. Rev. B 95 235-13 